\title{
ON MIXED TWO-TERM EXPONENTIAL SUMS
}

\author{
ZHANG TIANPING
}

ABSTRACT. In this paper, we shall use analytic methods to study the hybrid mean value involving the mixed two-term exponential sums $C(m, n, r$ $\chi ; q)$, and give several sharp asymptotic formulae.

\section{Introduction}

The classical two-term exponential sums is defined as

$$
C(m, n, r ; q)=\sum_{a=1}^{q} e\left(\frac{m a^{r}+n a}{q}\right),
$$

where $e(y)=e^{2 \pi i y}, q, m, n, r$ are integers with $q, r \geq 2$.

This summation is very important in additive number theory, because there exists close connections between this summation and Waring's problem. Many scholars have studied its arithmetical properties. For example, Davenport and Heilbronn [7] proved that

$$
C\left(m, n, r ; p^{\alpha}\right) \ll_{r} p^{\theta \alpha}\left(n, p^{\alpha}\right), \quad \text { if } p \nmid m,
$$

where $\theta=2 / 3$ if $r=3$, and $\theta=3 / 4$ if $r \geq 3$. Applying Weil's estimate for exponential sums over finite fields, Hua [8] proved that $\theta=1 / 2$ for all $r \geq 2$. Improvements have been made by Smith [17], Loxton and Smith [13], Loxton and Vaughan [14], Dabrowski and Fisher [5], etc. Recently, Ye found certain identities between the two-term exponential sums and hyper-Kloosterman sums (see Theorem 3 of reference [20]), these identities are in turn deduced from generalized Davenport-Hasse identities of Gauss sums. Applying the better bounds for hyper-Kloosterman sums for prime power modulus obtained in [5], he established better bounds for it.

Received October 31, 2008; Revised August 27, 2009

2000 Mathematics Subject Classification. 11L05.

Key words and phrases. two-term exponential sums, Gauss sums, hybrid mean value.

This work is supported by the NSF (10671155, 10871123), the Fundamental Research Funds for the Central Universities (GK200902051), and the Pre-Research Foundation of Shaanxi Normal University (200902007). 
The mixed two-term exponential sum is defined as

$$
C(m, n, r, \chi ; q)=\sum_{a=1}^{q} \chi(a) e\left(\frac{m a^{r}+n a}{q}\right),
$$

where $\chi$ denotes a Dirichlet character modulo $q$. If $q=p$, it follows from Weil's work [18] that for all $\chi$ modulo $p$, and $p \nmid m$, we have

$$
|C(m, n, r, \chi ; p)| \leq r p^{\frac{1}{2}} .
$$

For $q=p^{\alpha}$, Cochrane and Zheng [3] showed the following upper bound

$$
\left|C\left(m, n, r, \chi ; p^{\alpha}\right)\right| \leq r p^{\frac{2}{3} \alpha}\left(n, p^{\alpha}\right)^{\frac{1}{3}},
$$

where $r \geq 2, \alpha \geq 1, p>2$ and $m, n$ are any integers with $p \nmid m$; While if $p=2$, then for all $\chi$ modulo $2^{\alpha}$, they showed

$$
\left|C\left(m, n, r, \chi ; 2^{\alpha}\right)\right| \leq 2 r 2^{\frac{2}{3} \alpha}\left(n, 2^{\alpha}\right)^{\frac{1}{3}}
$$

and proved that the exponent $\frac{2}{3} \alpha$ is the best possible. Under the additional assumption that $\chi$ is a multiplicative character modulo $p^{\alpha}$ of conductor $p$ and $p \nmid(m, n)$, they in [4] got the improved upper bound

$$
\left|C\left(m, n, r, \chi ; p^{\alpha}\right)\right| \leq r p^{\frac{\alpha}{2}} .
$$

In [19] Xu, Zhang, and Zhang studied the asymptotic properties of the $2 l$-th power mean

$$
\sum_{\chi \bmod q} \sum_{m=1}^{q}|C(m, n, \chi ; q)|^{2 l}
$$

and gave the following exact formula in the case $l=2$.

Proposition. Let $q \geq 3$ be an integer and $k$ be any positive integer with $(k, q)=$ 1. Then for any fixed integer $n$ with $(n, q)=1$, we have the identity

$$
\begin{aligned}
& \sum_{m=1 \chi}^{q} \sum_{\substack{\bmod q \\
=}}|C(m, n, k, \chi ; q)|^{4} \\
= & q \phi^{3}(q) d(q) \prod_{\substack{p^{\alpha} \| q \\
\alpha \geq 2}}\left(1-\frac{2(k, p-1)-1}{(\alpha+1)(p-1)}\right) \prod_{p \| q}\left(1-\frac{2(k, p-1)-1}{2(p-1)}+\frac{(k, p-1)^{2}-1}{2(p-1)^{2}}\right),
\end{aligned}
$$

where $\phi(q)$ is the Euler function, $d(n)$ is the divisor function, $\prod_{p^{\alpha} \| q}$ denotes the product over all $p$ such that $p^{\alpha} \mid q$ and $p^{\alpha+1} \nmid q$.

If we assume that $k+1 \equiv 0(\bmod \phi(q))$, the mixed two-term exponential sum is the general Kloosterman sum $S(m, n, \chi ; q)$ which is defined as follows

$$
S(m, n, \chi ; q)=\sum_{a=1}^{q} \chi(a) e\left(\frac{m a+n \bar{a}}{q}\right),
$$


where $a \bar{a} \equiv 1(\bmod q)$. Chowla [2] and Malyshev [15] proved that for any integers $m$ and $n$, we have

$$
|S(m, n, \chi ; p)| \ll(m, n, p)^{1 / 2} p^{1 / 2+\epsilon},
$$

where $\epsilon$ is any positive real number. But about the properties of $S(m, n, \chi ; q)$ for general $q$, we know very little about it so far. In fact, the value of $\mid S(m, n, \chi$; $q) \mid$ is quite irregular if $q$ is not a prime. However, Zhang (see [22] and [23]) showed that the values of $S(m, n, \chi ; q)$ enjoy many good distribution properties by proving that

$$
\sum_{\chi \bmod q} \sum_{m=1}^{q}|S(m, n, \chi ; q)|^{4}=q \phi^{3}(q) d(q) \prod_{p^{\alpha} \| q}\left(1-\frac{1}{(\alpha+1)(p-1)}\right)
$$

and

$$
\sum_{m=1}^{p}|S(m, n, \chi ; p)|^{4}=\left\{\begin{aligned}
p\left(2 p^{2}-3 p-3\right), & \text { if } \chi \text { is the principal character } \bmod p ; \\
p^{2}(3 p-7), & \text { if } \chi \text { is the Legendre symbol; } \\
2 p^{2}(p-3), & \text { if } \chi \text { is a complex character mod } p .
\end{aligned}\right.
$$

He [24] also studied the hybrid mean value between Dirichlet $L$-functions and the general Kloosterman sums, and obtained that

$$
\begin{aligned}
& \sum_{\substack{\chi \bmod q \\
\chi \neq \chi_{0}}}|S(m, n, \chi ; q)|^{2}|L(1, \chi)|^{2 k} \\
= & \left(\frac{\pi^{2}}{6}\right)^{2 k-1} \phi^{2}(q) \prod_{p \mid q}\left(1-\frac{1}{p^{2}}\right)^{2 k-1} \prod_{p \nmid q}\left(1-\frac{1-\left(\begin{array}{c}
2 k-2 \\
k-1
\end{array}\right)}{p^{2}}\right)+O\left(q^{\frac{3}{2}+\epsilon}\right) .
\end{aligned}
$$

Moreover, Liu and Zhang [12] investigated the hybrid mean value involving the general Kloosterman sums further, and obtained that for any positive integer $k$ and integers $m$ and $n$ with $(m n, q)=1$, we have

$$
\sum_{\substack{\chi \bmod q \\ \chi \neq \chi_{0}}}|S(m, n, \chi ; q)|^{2}\left|\frac{L^{\prime}}{L}(1, \chi)\right|^{2 k}=A(k, q) \phi^{2}(q)+O\left(q^{\frac{3}{2}+\epsilon}\right),
$$

where $A(k, q)=\sum_{\substack{n=1 \\(n, q)=1}}^{+\infty} \frac{\tau_{k}^{2}(n)}{n^{2}}$ is a constant depending on $k$ and $q$,

$$
\tau_{k}(n)=\sum_{m_{1} m_{2} \cdots m_{k}=n} \Lambda\left(m_{1}\right) \Lambda\left(m_{2}\right) \cdots \Lambda\left(m_{k}\right),
$$

and $\Lambda(n)$ is the Mangoldt function.

It might be interesting to consider similar mean values on the mixed twoterm exponential sums. In this paper, we shall use analytic methods to study the hybrid mean value involving the mixed two-term exponential sums $C(m, n$, 
$r, \chi ; q)$, and give several sharp asymptotic formulae. That is, we shall prove the following:

Theorem 1. Let $p$ be an odd prime, $n, r, \alpha$ be integers with $r, \alpha \geq 2, p \nmid r(r-$ $1), p \nmid n$ and $(r-1, p-1)=1$. Then for any integers $m$ and $k \geq 1$, we have

$$
\sum_{\chi \bmod _{\chi \neq \chi_{0}} p^{\alpha}}\left|C\left(m, n, r, \chi ; p^{\alpha}\right)\right|^{2}\left|\frac{L^{\prime}}{L}(1, \chi)\right|^{2 k}=A\left(k, p^{\alpha}\right) p^{2 \alpha}+O\left(p^{\frac{3 \alpha}{2}+\epsilon}\right) .
$$

Theorem 2. Let $p$ be an odd prime, $n, r, \alpha$ be integers with $r, \alpha \geq 2, p \nmid r(r-$ $1), p \nmid n$ and $(r-1, p-1)=1$. Then for any integers $m$ and $k \geq 2$, we have

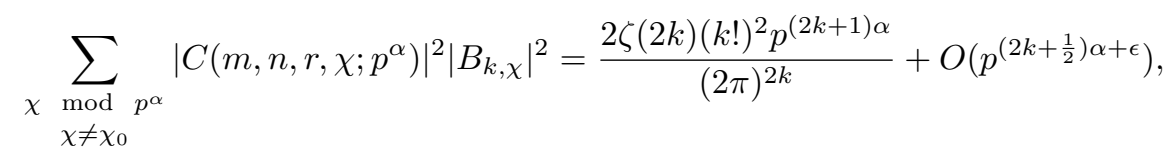

where $\zeta(s)$ is the Riemann zeta function and $B_{k, \chi}$ is a generalized Bernoulli numbers defined by

$$
\sum_{a=1}^{q} \chi(a) \frac{t e^{a t}}{e^{q t}-1}=\sum_{k=0}^{+\infty} \frac{B_{k, \chi}}{k !} t^{k}
$$

Theorem 3. Let $p$ be an odd prime, $n, r, \alpha$ be integers with $r, \alpha \geq 2, p \nmid r(r-$ $1), p \nmid n$ and $(r-1, p-1)=1$. Then for any integers $m$ and $k \geq 2$, we have

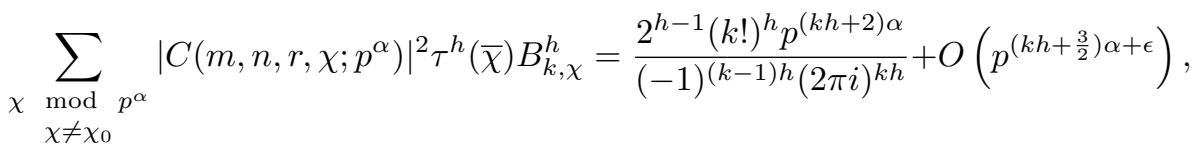
where $\tau(\chi)$ is Gauss sums.

Remark. In Theorem 1, if we bound the left hand side by estimation of individual terms, we can use the bounds $\left|C\left(m, n, r, \chi ; p^{\alpha}\right)\right|=O\left(p^{\frac{2}{3} \alpha+\epsilon}\right)$ and $\left|\frac{L^{\prime}}{L}(1, \chi)\right|=O\left(p^{\epsilon}\right)$. This will give us a bound for the left hand side $O\left(p^{\frac{7}{3} \alpha+\epsilon}\right)$, which is much worse than the bound of the right hand side. Similar discussions can be made in Theorems 2 and 3 .

\section{Several lemmas}

To complete the proof of the theorems, we need the following several lemmas.

Lemma 1. Let $p$ be an odd prime, $n, r, \alpha$ be integers with $r, \alpha \geq 2, p \nmid r(r-$ $1), p \nmid n$ and $(r-1, p-1)=1$. Then for any integer $m$, we have the following sharp bound that

$$
\left|C\left(m, n, r ; p^{\alpha}\right)\right| \leq p^{\frac{\alpha}{2}} .
$$

Proof. This is Corollary 1 of [3]. 
Lemma 2. Let $m, n, r, q$ be integers $r \geq 2$ and $q \geq 3$. Then we have the identity

$$
|C(m, n, r, \chi ; q)|^{2}=\phi(q)+\sum_{a=2}^{q} \chi(a) \sum_{b=1}^{q} e\left(\frac{m b^{r}\left(a^{r}-1\right)+n b(a-1)}{q}\right) .
$$

Proof. From the properties of residue systems, we have

$$
\begin{aligned}
|C(m, n, r, \chi ; q)|^{2} & =\sum_{a=1}^{q} \sum_{b=1}^{q} \chi(a \bar{b}) e\left(\frac{m\left(a^{r}-b^{r}\right)+n(a-b)}{q}\right) \\
& =\sum_{a=1}^{q} \chi(a) \sum_{b=1}^{\prime} e\left(\frac{m b^{r}\left(a^{r}-1\right)+n b(a-1)}{q}\right) \\
& =\phi(q)+\sum_{a=2}^{\prime} \chi(a) \sum_{b=1}^{q} e\left(\frac{m b^{r}\left(a^{r}-1\right)+n b(a-1)}{q}\right) .
\end{aligned}
$$

This completes the proof.

Lemma 3. For any positive integer $k$, we have the asymptotic formula that

$$
\sum_{\substack{\chi \bmod _{\chi} \\ \chi \neq \chi_{0}}}\left|\frac{L^{\prime}}{L}(1, \chi)\right|^{2 k}=A(k, q) \phi(q)+O\left(q^{\epsilon}\right) .
$$

Proof. This is Theorem 2.1 of [12].

Lemma 4. Let $p$ be an odd prime, $n, r, \alpha$ be integers with $r, \alpha \geq 2, p \nmid r(r-$ $1), p \nmid n$ and $(r-1, p-1)=1$. Then for any integers $m$ and $k \geq 1$, we have the estimate that

$$
\sum_{\substack{\chi \neq \chi_{0} \\ \chi \neq \chi_{0}}}\left[\sum_{a=2}^{p^{\alpha}} \chi(a) \sum_{b=1}^{p^{\alpha}} e\left(\frac{m b^{r}\left(a^{r}-1\right)+n b(a-1)}{p^{\alpha}}\right)\right]\left|\frac{L^{\prime}}{L}(1, \chi)\right|^{2 k} \ll p^{\frac{3 \alpha}{2}+\epsilon} .
$$

Proof. Let $\chi_{1}$ be the non-principal real character modulo $p^{\alpha}$. Then from Siegel's theorem [16] and the properties of $L$-functions we know that

$$
\frac{L^{\prime}}{L}\left(1, \chi_{1}\right) \ll \frac{p^{\alpha \epsilon}\left(\log p^{\alpha}\right)^{2}}{C_{1}(\epsilon)} .
$$

For any complex character $\chi$ modulo $p^{\alpha}$, and $p^{\alpha} \leq \exp \left[C^{\prime}(\log x)^{\frac{1}{2}}\right]$, where $C^{\prime}$ is any positive constant, from [6] we have

$$
\Psi(x, \chi)=\sum_{n \leq x} \chi(n) \Lambda(n) \ll x \exp \left(-C^{\prime \prime}(\log x)^{\frac{1}{2}}\right)
$$


holds for some positive $C^{\prime \prime}$ depending only on $C^{\prime}$. Then for parameter $N \geq$ $\exp \left(\frac{\left(\log p^{\alpha}\right)^{2}}{\left(C^{\prime}\right)^{2}}\right)$, by Abel's identity we can get

$$
\begin{aligned}
\frac{L^{\prime}}{L}(1, \chi) & =\sum_{n=1}^{+\infty} \frac{\chi(n) \Lambda(n)}{n} \\
& =\sum_{1 \leq n \leq N} \frac{\chi(n) \Lambda(n)}{n}+\int_{N}^{+\infty} \frac{\sum_{N<n \leq y} \chi(n) \Lambda(n)}{y^{2}} \mathrm{~d} y \\
& =\sum_{1 \leq n \leq N} \frac{\chi(n) \Lambda(n)}{n}+O\left(\frac{\log N}{\exp \left(C^{\prime \prime}(\log N)^{\frac{1}{2}}\right)}\right) .
\end{aligned}
$$

Therefore we have

$$
\begin{aligned}
& \sum_{\substack{\chi \neq \chi_{0} \\
\chi \neq \chi_{0}}}\left[\sum_{a=2}^{p^{\alpha}} \chi(a) \sum_{b=1}^{p^{\alpha}} e\left(\frac{m b^{r}\left(a^{r}-1\right)+n b(a-1)}{p^{\alpha}}\right)\right]\left|\frac{L^{\prime}}{L}(1, \chi)\right|^{2 k} \\
& =\sum_{\substack{\chi \bmod p^{\alpha} \\
\chi \neq \chi_{0} \\
\chi \neq \chi_{1}}}\left[\sum_{a=2}^{p^{\alpha}} \chi(a) \sum_{b=1}^{p^{\alpha}} e\left(\frac{m b^{r}\left(a^{r}-1\right)+n b(a-1)}{p^{\alpha}}\right)\right]\left|\frac{L^{\prime}}{L}(1, \chi)\right|^{2 k} \\
& +O\left(\frac{p^{\frac{3 \alpha}{2}} p^{2 k \alpha \epsilon}\left(\log p^{\alpha}\right)^{4 k}}{C_{1}^{2 k}(\epsilon)}\right) \\
& =\sum_{\substack{\chi \bmod p^{\alpha} \\
\chi \neq \chi_{0} \\
\chi \neq \chi_{1}}}\left[\sum_{a=2}^{p^{\alpha}} \chi(a) \sum_{b=1}^{p^{\alpha}} e\left(\frac{m b^{r}\left(a^{r}-1\right)+n b(a-1)}{p^{\alpha}}\right)\right]\left|\sum_{1 \leq l \leq N} \frac{\chi(l) \Lambda(l)}{l}\right|^{2 k} \\
& +O\left(\frac{p^{\frac{5 \alpha}{2}} \log ^{2 k} N}{\exp \left(C^{\prime \prime}(\log N)^{\frac{1}{2}}\right)}\right)+O\left(\frac{p^{\frac{3 \alpha}{2}} p^{2 k \alpha \epsilon}\left(\log p^{\alpha}\right)^{4 k}}{C_{1}^{2 k}(\epsilon)}\right) \\
& =\sum_{\chi \bmod p^{\alpha}}\left[\sum_{a=2}^{p^{\alpha}} \chi(a) \sum_{b=1}^{p^{\alpha}} e\left(\frac{m b^{r}\left(a^{r}-1\right)+n b(a-1)}{p^{\alpha}}\right)\right]\left|\sum_{1 \leq l \leq N} \frac{\chi(l) \Lambda(l)}{l}\right|^{2 k} \\
& +O\left(p^{\frac{3 \alpha}{2}} \log ^{2 k} N\right)+O\left(\frac{p^{\frac{5 \alpha}{2}} \log ^{2 k} N}{\exp \left(C^{\prime \prime}(\log N)^{\frac{1}{2}}\right)}\right)+O\left(\frac{p^{\frac{3 \alpha}{2}} p^{2 k \alpha \epsilon}\left(\log p^{\alpha}\right)^{4 k}}{C_{1}^{2 k}(\epsilon)}\right) .
\end{aligned}
$$


Then from the orthogonality relations for character sums and Lemma 1, we can get

$$
\begin{aligned}
& \sum_{\chi \bmod p^{\alpha}}\left[\sum_{a=2}^{p^{\alpha}} \chi(a) \sum_{b=1}^{p^{\alpha}} e\left(\frac{m b^{r}\left(a^{r}-1\right)+n b(a-1)}{p^{\alpha}}\right)\right]\left|\sum_{1 \leq l \leq N} \frac{\chi(l) \Lambda(l)}{l}\right|^{2 k} \\
& =\sum_{\chi \bmod p^{\alpha}}\left[\sum_{a=2}^{p^{\alpha}} \chi(a) \sum_{b=1}^{p^{\alpha}} e\left(\frac{m b^{r}\left(a^{r}-1\right)+n b(a-1)}{p^{\alpha}}\right)\right]\left|\sum_{1 \leq l \leq N^{k}} \frac{\chi(l) \tau_{k}(l)}{l}\right|^{2} \\
& =\sum_{a=2}^{p^{\alpha}} \sum_{b=1}^{p^{\alpha}} e\left(\frac{m b^{r}\left(a^{r}-1\right)+n b(a-1)}{p^{\alpha}}\right) \\
& \times \sum_{1 \leq n_{1} \leq N^{k}} \sum_{1 \leq n_{2} \leq N^{k}} \frac{\tau_{k}\left(n_{1}\right) \tau_{k}\left(n_{2}\right)}{n_{1} n_{2}} \sum_{\chi \bmod p^{\alpha}} \chi\left(a n_{1}\right) \bar{\chi}\left(n_{2}\right) \\
& \leq \phi\left(p^{\alpha}\right) \sum_{a=2}^{p^{\alpha}}\left|C\left(m, n, r ; p^{\alpha}\right)\right| \sum_{\substack{1 \leq n_{1} \leq N^{k} \\
a n_{1} \equiv n_{2} \bmod p^{\alpha}}} \sum_{\substack{1 \leq n_{2} \leq N^{k} \\
\operatorname{mos}}} \frac{\tau_{k}\left(n_{1}\right) \tau_{k}\left(n_{2}\right)}{n_{1} n_{2}}
\end{aligned}
$$

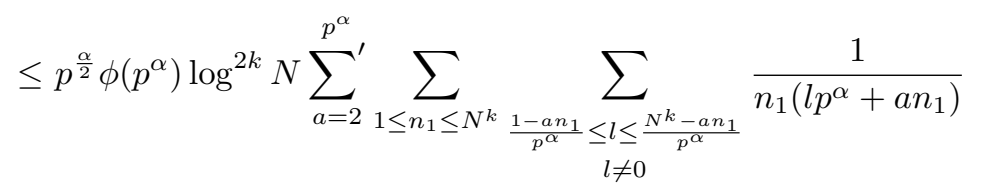

$$
\begin{aligned}
& +p^{\frac{\alpha}{2}} \phi\left(p^{\alpha}\right) \log ^{2 k} N \sum_{a=2}^{p^{\alpha}} \sum_{1 \leq n_{1} \leq N^{k}} \frac{1}{a n_{1}^{2}} \\
& \ll p^{\frac{\alpha}{2}} \phi\left(p^{\alpha}\right) \log ^{2 k} N \sum_{a=2}^{p^{\alpha}} \frac{1}{a}+p^{\frac{\alpha}{2}} \log ^{2 k+2} N \sum_{a=2}^{p^{\alpha}} 1 \\
& \ll p^{\frac{\alpha}{2}} \phi\left(p^{\alpha}\right) \log ^{2 k+2} N \text {. }
\end{aligned}
$$

Taking $N=\max \left\{\exp \left(\frac{\log ^{2} p^{\alpha}}{\left(C^{\prime}\right)^{2}}\right), \exp \left(\frac{\log ^{2} p^{\alpha}}{\left(C^{\prime \prime}\right)^{2}}\right)\right\}$ in the above, we may immediately get

$$
\sum_{\substack{\chi \bmod p^{\alpha} \\ \chi \neq \chi_{0}}}\left[\sum_{a=2}^{p^{\alpha}} \chi(a) \sum_{b=1}^{p^{\alpha}} e\left(\frac{m b^{r}\left(a^{r}-1\right)+n b(a-1)}{p^{\alpha}}\right)\right]\left|\frac{L^{\prime}}{L}(1, \chi)\right|^{2 k} \ll p^{\frac{3 \alpha}{2}+\epsilon}
$$

This completes the proof. 
Lemma 5. Let $q \geq 3$ be an integer. Then for any positive integer $k \geq 2$, we have

$$
\sum_{\substack{\chi \neq \chi_{0} \\ r_{1}=-\infty}} \sum_{\substack{r_{2}=-\infty \\ r_{1} \neq 0}}^{+\infty} \frac{G\left(r_{1}, \chi\right) \overline{G\left(r_{2}, \chi\right)}}{\left(r_{1} r_{2}\right)^{k}}=2 \zeta(2 k) \phi^{2}(q)+O\left(q^{1+\epsilon}\right) .
$$

Proof. This is Lemma 2.1 of [11].

Lemma 6. Let $p$ be an odd prime, $n, r, \alpha$ be integers with $r, \alpha \geq 2, p \nmid r(r-$ $1), p \nmid n$ and $(r-1, p-1)=1$. Then for any integers $m$ and $k \geq 2$, we have

$$
\begin{aligned}
\Phi= & \sum_{a=2}^{p^{\alpha}} \sum_{b=1}^{p^{\alpha}} e\left(\frac{m b^{r}\left(a^{r}-1\right)+n b(a-1)}{p^{\alpha}}\right) \\
& \times \sum_{\substack{r_{1}=-\infty \\
r_{1} \neq 0}}^{+\infty} \sum_{\substack{r_{2}=-\infty \\
r_{2} \neq 0}}^{+\infty} \sum_{\substack{\bmod p^{\alpha} \\
\chi \neq \chi_{0}}} \frac{\chi(a) G\left(r_{1}, \chi\right) \overline{G\left(r_{2}, \chi\right)}}{\left(r_{1} r_{2}\right)^{k}} \\
& \ll p^{\frac{5 \alpha}{2}+\epsilon},
\end{aligned}
$$

where $G(r, \chi)=\sum_{b=1}^{p^{\alpha}} e\left(\frac{r b}{p^{\alpha}}\right)$.

Proof. From the orthogonality relation for character $\chi \bmod p^{\alpha}$, we have

$$
\begin{aligned}
& \Phi=\sum_{a=2}^{p^{\alpha}} \sum_{b=1}^{p^{\alpha}} e\left(\frac{m b^{r}\left(a^{r}-1\right)+n b(a-1)}{p^{\alpha}}\right) \\
& \times \sum_{\substack{r_{1}=-\infty \\
r_{1} \neq 0}}^{+\infty} \sum_{\substack{r_{2}=-\infty \\
r_{2} \neq 0}}^{+\infty} \sum_{\substack{\bmod p^{\alpha} \\
\chi \neq \chi_{0}}} \frac{\chi(a) G\left(r_{1}, \chi\right) \overline{G\left(r_{2}, \chi\right)}}{\left(r_{1} r_{2}\right)^{k}} \\
& =\sum_{a=2}^{p^{\alpha}} \sum_{b=1}^{p^{\alpha}} e\left(\frac{m b^{r}\left(a^{r}-1\right)+n b(a-1)}{p^{\alpha}}\right) \sum_{\substack{r_{1}=-\infty \\
r_{1} \neq 0}}^{+\infty} \sum_{\substack{r_{2}=-\infty \\
r_{2} \neq 0}}^{+\infty} \frac{1}{\left(r_{1} r_{2}\right)^{k}} \\
& \times \sum_{\chi \bmod } \chi(a s \bar{t}) \sum_{p^{\alpha}}^{p^{\alpha}} \sum_{t=1}^{p^{\alpha}} e\left(\frac{s r_{1}-t r_{2}}{p^{\alpha}}\right) \\
& -\sum_{a=2}^{p^{\alpha}} \sum_{b=1}^{p^{\alpha}} e\left(\frac{m b^{r}\left(a^{r}-1\right)+n b(a-1)}{p^{\alpha}}\right)\left(\sum_{\substack{t=-\infty \\
t \neq 0}}^{+\infty} \frac{C_{p^{\alpha}}(t)}{t^{k}}\right)^{2} \\
& =\phi\left(p^{\alpha}\right) \sum_{a=2}^{p^{\alpha}} \sum_{b=1}^{p^{\alpha}} e\left(\frac{m b^{r}\left(a^{r}-1\right)+n b(a-1)}{p^{\alpha}}\right) \sum_{\substack{r_{1}=-\infty \\
r_{1} \neq 0}}^{+\infty} \sum_{\substack{r_{2}=-\infty \\
r_{2} \neq 0}}^{+\infty} \frac{C_{p^{\alpha}}\left(r_{1}-a r_{2}\right)}{\left(r_{1} r_{2}\right)^{k}}
\end{aligned}
$$




$$
-\sum_{a=2}^{p^{\alpha}} \sum_{b=1}^{p^{\alpha}} e\left(\frac{m b^{r}\left(a^{r}-1\right)+n b(a-1)}{p^{\alpha}}\right)\left(\sum_{\substack{t=-\infty \\ t \neq 0}}^{+\infty} \frac{C_{p^{\alpha}}(t)}{t^{k}}\right)^{2},
$$

where $C_{p^{\alpha}}(t)=G\left(t, \chi_{0}\right)=\sum_{a=1}^{\prime p^{\alpha}} e\left(\frac{t a}{p^{\alpha}}\right)$ is Ramanujan sums. Then from the identity that

$$
C_{p^{\alpha}}(t)=\sum_{d \mid\left(p^{\alpha}, t\right)} d \mu\left(\frac{p^{\alpha}}{d}\right)
$$

we may have

$$
\begin{aligned}
& \sum_{a=2}^{p^{\alpha}} \sum_{b=1}^{p^{\alpha}} e\left(\frac{m b^{r}\left(a^{r}-1\right)+n b(a-1)}{p^{\alpha}}\right) \sum_{\substack{r_{1}=-\infty \\
r_{1} \neq 0}}^{+\infty} \sum_{\substack{r_{2}=-\infty \\
r_{2} \neq 0}}^{+\infty} \frac{C_{p^{\alpha}}\left(r_{1}-a r_{2}\right)}{\left(r_{1} r_{2}\right)^{k}} \\
& =\sum_{a=2}^{p^{\alpha}} \sum_{b=1}^{p^{\alpha}}{ }^{\prime} e\left(\frac{m b^{r}\left(a^{r}-1\right)+n b(a-1)}{p^{\alpha}}\right) \\
& \times \sum_{\substack{r_{1}=-\infty \\
r_{1} \neq 0}}^{+\infty} \sum_{\substack{r_{2}=-\infty \\
r_{2} \neq 0}}^{+\infty} \frac{1}{\left(r_{1} r_{2}\right)^{k}} \sum_{\substack{d \mid\left(p^{\alpha}, r_{1}-a r_{2}\right) \\
p_{2}}} d \mu\left(\frac{p^{\alpha}}{d}\right) \\
& \leq p^{\frac{\alpha}{2}} \sum_{d \mid p^{\alpha}} d \sum_{a=2}^{p^{\alpha}} \sum_{\substack{r_{1}=-\infty \\
r_{1} \neq 0 \\
r_{1} \equiv a r_{2}}}^{+\infty} \sum_{\substack{r_{2}=-\infty \\
r_{2} \neq 0}}^{+\infty} \frac{1}{\left(r_{1} r_{2}\right)^{k}}
\end{aligned}
$$

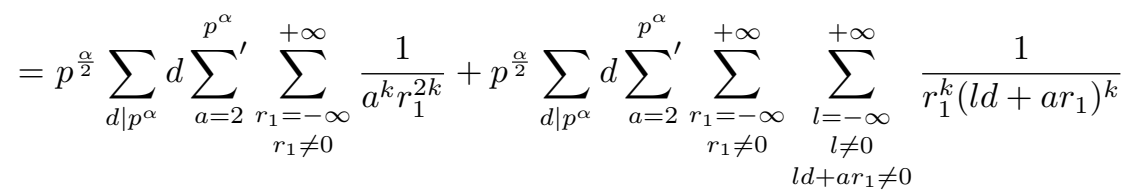

$$
\begin{aligned}
& \ll p^{\frac{3 \alpha}{2}+\epsilon} .
\end{aligned}
$$

Similarly, we can obtain

$$
\sum_{a=2}^{p^{\alpha}} \sum_{b=1}^{p^{\alpha}} e\left(\frac{m b^{r}\left(a^{r}-1\right)+n b(a-1)}{p^{\alpha}}\right)\left(\sum_{\substack{t=-\infty \\ t \neq 0}}^{+\infty} \frac{C_{p^{\alpha}}(t)}{t^{k}}\right)^{2} \ll p^{\frac{3 \alpha}{2}+\epsilon} .
$$

Thus we have

$$
\Phi \ll p^{\frac{5 \alpha}{2}+\epsilon} .
$$

This completes the proof. 
Lemma 7. Let $q$ and $r$ be integers with $q \geq 2$ and $(r, q)=1, \chi$ be a Dirichlet character modulo $q$. Then we have the identities

$$
\sum_{\chi \bmod q}^{*} \chi(r)=\sum_{d \mid(q, r-1)} \mu\left(\frac{q}{d}\right) \phi(d)
$$

and

$$
J(q)=\sum_{d \mid q} \mu(d) \phi\left(\frac{q}{d}\right)
$$

where $\sum^{*} \chi \bmod q$ denotes the summation over all primitive characters modulo $q$ and $J(q)$ denotes the number of primitive characters modulo $q$.

Proof. This is Lemma 3 of [21].

Lemma 8. Let $p$ be an odd prime and $\alpha$ be an integer with $\alpha \geq 2$. Then for any non-primitive character $\chi$ modulo $p^{\alpha}$, we have the identity that

$$
\tau(\chi)=\sum_{a=1}^{p^{\alpha}} \chi(a) e\left(\frac{a}{p^{\alpha}}\right)=0 .
$$

Proof. This is Theorem 7.4.2 of [9].

Lemma 9. Let $p$ be an odd prime and $\alpha$ be an integer with $\alpha \geq 2$. Then for any positive integers $k$ and $h$, we have the asymptotic formula that

$$
\sum_{\chi \bmod p^{\alpha}}^{*} \tau^{h}(\bar{\chi}) B_{k, \chi}^{h}=\frac{2^{h-1}(k !)^{h} p^{(k h+1) \alpha}}{(-1)^{(k-1) h}(2 \pi i)^{k h}}+O\left(p^{k h \alpha+\epsilon}\right) .
$$

Proof. This can be easily deduced from the proof of Theorem in [10].

Lemma 10. Let $p$ be an odd prime, $n, r, \alpha$ be integers with $r, \alpha \geq 2, p \nmid r(r-$ $1), p \nmid n$ and $(r-1, p-1)=1$. Then for any integers $m$ and $k \geq 2$, we have the estimates

$$
\Psi_{1}=\sum_{a=2}^{p^{\alpha}} \sum_{b=1}^{p^{\alpha}} e\left(\frac{m b^{r}\left(a^{r}-1\right)+n b(a-1)}{p^{\alpha}}\right) \sum_{\substack{\chi \bmod p^{\alpha} \\ \chi(-1)=-1}} \chi(a) L^{h}(k, \bar{\chi}) \ll p^{\frac{3 \alpha}{2}+\epsilon}
$$

and

$$
\Psi_{2}=\sum_{a=2}^{p^{\alpha}} \sum_{b=1}^{p^{\alpha}} e\left(\frac{m b^{r}\left(a^{r}-1\right)+n b(a-1)}{p^{\alpha}}\right) \sum_{\substack{\chi \bmod \\ \chi(-1)=1}} \chi(a) L^{h}(k, \bar{\chi}) \ll p^{\frac{3 \alpha}{2}+\epsilon} .
$$

Proof. We begin with the estimate for $\Psi_{1}$. Let $d_{h}(r)$ be the $h$-divisor function (i.e., the number of positive integer solutions of the equation $t=t_{1} t_{2} \cdots t_{h}$ ). 
Then for any parameter $N \geq p^{\alpha}$ and non-principal character $\chi$ modulo $p^{\alpha}$, applying Abel's identity we have

$$
L^{h}(k, \bar{\chi})=\sum_{t=1}^{\infty} \frac{\bar{\chi}(t) d_{h}(t)}{t^{k}}=\sum_{1 \leq r \leq N} \frac{\bar{\chi}(t) d_{h}(t)}{t^{k}}+k \int_{N}^{\infty} \frac{A(y, \bar{\chi})}{y^{k+1}} \mathrm{~d} y
$$

where $A(y, \bar{\chi})=\sum_{N<t \leq y} \bar{\chi}(t) d_{h}(t)$. Applying Lemma 4 in [24] and Cauchy inequality, we have

$$
\sum_{\substack{\chi \bmod p^{\alpha} \\ \chi(-1)=-1}}|A(y, \bar{\chi})| \leq\left(\phi\left(p^{\alpha}\right) \sum_{\substack{\chi \bmod p^{\alpha} \\ \chi(-1)=-1}}|A(y, \bar{\chi})|^{2}\right)^{1 / 2} \ll y^{1-\left(1 / 2^{h-1}\right)+\epsilon} \phi^{\frac{3}{2}}\left(p^{\alpha}\right) .
$$

Hence we have

$$
\begin{aligned}
& \quad \sum_{a=2}^{p^{\alpha}} \sum_{b=1}^{p^{\alpha}} e\left(\frac{m b^{r}\left(a^{r}-1\right)+n b(a-1)}{p^{\alpha}}\right) \sum_{\substack{\chi \bmod p^{\alpha} \\
\chi(-1)=-1}}^{*} \chi(a) k \int_{N}^{\infty} \frac{A(y, \bar{\chi})}{y^{k+1}} d y \\
& \ll p^{\frac{3 \alpha}{2}} \int_{N}^{\infty} \frac{k}{y^{k+1}}\left(\sum_{\substack{\chi \bmod p^{\alpha} \\
\chi(-1)=-1}}|A(y, \bar{\chi})|\right) d y \ll p^{\frac{3 \alpha}{2}} \int_{N}^{\infty} \frac{y^{1-\frac{1}{2^{h-1}}+\epsilon} \phi^{\frac{3}{2}}\left(p^{\alpha}\right)}{y^{2}} d y \\
& \ll \frac{p^{3 \alpha+\epsilon}}{N^{2^{1-h}}} .
\end{aligned}
$$

Combining the above we can get

$$
\begin{aligned}
\Psi_{1}= & \sum_{a=2}^{p^{\alpha}} \sum_{b=1}^{p^{\alpha}} \sum^{\prime} e\left(\frac{m b^{r}\left(a^{r}-1\right)+n b(a-1)}{p^{\alpha}}\right) \sum_{1 \leq t \leq N} \frac{d_{h}(t)}{t} \sum_{\substack{\chi \bmod p^{\alpha} \\
\chi(-1)=-1}}^{*} \bar{\chi}(t) \chi(a) \\
& +O\left(\frac{p^{3 \alpha+\epsilon}}{N^{2^{1-h}}}\right) .
\end{aligned}
$$

Note that for any integer $a$ with $(a, q)=1$, from Lemma 7 we have

$$
\begin{aligned}
\sum_{\substack{\chi \bmod q \\
\chi(-1)=-1}}^{*} \chi(a) & =\frac{1}{2} \sum_{\chi} \sum_{\bmod q}^{*}(1-\chi(-1)) \chi(a) \\
& =\frac{1}{2} \sum_{\chi}^{*} \chi(a)-\frac{1}{2} \sum_{\chi \bmod q}^{*} \chi(-a) \\
& =\frac{1}{2} \sum_{s \mid(q, a-1)} \mu\left(\frac{q}{s}\right) \phi(s)-\frac{1}{2} \sum_{s \mid(q, a+1)} \mu\left(\frac{q}{s}\right) \phi(s) .
\end{aligned}
$$


Therefore we have

$$
\begin{aligned}
& \Psi_{1} \\
& =\frac{1}{2} \sum_{a=2}^{p^{\alpha}} \sum_{b=1}^{p^{\alpha}} e\left(\frac{m b^{r}\left(a^{r}-1\right)+n b(a-1)}{p^{\alpha}}\right) \sum_{\substack{1 \leq t \leq N \\
t}} \frac{d_{h}(t)}{t} \sum_{\substack{s \mid p^{\alpha} \\
t \equiv a \bmod s}} \mu\left(\frac{p^{\alpha}}{s}\right) \phi(s) \\
& -\frac{1}{2} \sum_{a=2}^{p^{\alpha}} \sum_{b=1}^{p^{\alpha}} e\left(\frac{m b^{r}\left(a^{r}-1\right)+n b(a-1)}{p^{\alpha}}\right) \\
& \times \sum_{1 \leq t \leq N} \frac{d_{h}(t)}{t} \sum_{\substack{s \mid p^{\alpha} \\
t \equiv-a \bmod s}} \mu\left(\frac{p^{\alpha}}{s}\right) \phi(s)+O\left(\frac{p^{3 \alpha+\epsilon}}{N^{2^{1-h}}}\right) \\
& =\frac{1}{2} \sum_{a=2}^{p^{\alpha}} \sum_{b=1}^{p^{\alpha}} e\left(\frac{m b^{r}\left(a^{r}-1\right)+n b(a-1)}{p^{\alpha}}\right) \frac{d_{h}(a)}{a} \sum_{s \mid p^{\alpha}} \mu\left(\frac{p^{\alpha}}{s}\right) \phi(s) \\
& +O\left(\frac{p^{3 \alpha+\epsilon}}{N^{2^{1-h}}}\right) \\
& +O\left(\sum_{a=2}^{p^{\alpha}} \sum_{b=1}^{p^{\alpha}} e\left(\frac{m b^{r}\left(a^{r}-1\right)+n b(a-1)}{p^{\alpha}}\right) \sum_{s \mid p^{\alpha}} \phi(s) \sum_{\frac{1-a}{s} \leq l \leq \frac{N}{s}}(l s+a)^{\epsilon-1}\right) \\
& +O\left(\sum_{a=2}^{p^{\alpha}} \sum_{b=1}^{p^{\alpha}} e\left(\frac{m b^{r}\left(a^{r}-1\right)+n b(a-1)}{p^{\alpha}}\right) \sum_{s \mid p^{\alpha}} \phi(s) \sum_{\frac{1+a}{s} \leq l \leq \frac{N}{s}}(l s-a)^{\epsilon-1}\right) \\
& =p^{\frac{3 \alpha}{2}+\epsilon}+N^{\epsilon}+O\left(\frac{p^{3 \alpha+\epsilon}}{N^{2^{1-h}}}\right) \text {, }
\end{aligned}
$$

where we have used the estimate $d_{h}(t) \ll t^{\epsilon}$.

Now taking $N=p^{3 \alpha \cdot 2^{h-2}}$ in the above, we may immediately obtain the following

$$
\Psi_{1} \ll p^{\frac{3 \alpha}{2}+\epsilon} .
$$

Using the similar method, we can obtain the second estimate. This completes the proof.

\section{Proof of the theorems}

In this section, we shall complete the proof of the theorems. First from Lemma 2 we have

$$
\sum_{\substack{\chi \bmod _{q} \\ \chi \neq \chi_{0}}}|C(m, n, r, \chi ; q)|^{2}\left|\frac{L^{\prime}}{L}(1, \chi)\right|^{2 k}
$$




$$
\begin{aligned}
=\phi(q) \sum_{\substack{\chi \bmod q \\
\chi \neq \chi_{0}}}\left|\frac{L^{\prime}}{L}(1, \chi)\right|^{2 k} \\
+\sum_{\substack{\chi \bmod q \\
\chi \neq \chi_{0}}}\left[\sum_{a=2}^{q} \chi(a) \sum_{b=1}^{\prime} e\left(\frac{m b^{r}\left(a^{r}-1\right)+n b(a-1)}{q}\right)\right]\left|\frac{L^{\prime}}{L}(1, \chi)\right|^{2 k} .
\end{aligned}
$$

Then from Lemmas 3 and 4, we may have

$$
\sum_{\substack{\bmod p^{\alpha} \\ \chi \neq \chi_{0}}}\left|C\left(m, n, r, \chi ; p^{\alpha}\right)\right|^{2}\left|\frac{L^{\prime}}{L}(1, \chi)\right|^{2 k}=A\left(k, p^{\alpha}\right) p^{2 \alpha}+O\left(p^{\frac{3 \alpha}{2}+\epsilon}\right) .
$$

This completes the proof of Theorem 1 .

Then we come to prove Theorem 2 . Let $q \geq 3$ be an integer. Then for any character $\chi$ modulo $q$, the generalized Bernoulli numbers can be expressed in terms of Bernoulli polynomials as

$$
B_{k, \chi}=q^{k-1} \sum_{a=1}^{q} \chi(a) B_{k}\left(\frac{a}{q}\right) .
$$

From Theorem 12.19 of [1] we know that if $0<x \leq 1$, then we have

$$
B_{k}(x)=-\frac{k !}{(2 \pi i)^{k}} \sum_{\substack{t=-\infty \\ t \neq 0}}^{+\infty} \frac{e(t x)}{t^{k}} .
$$

So we have

$$
B_{k, \chi}=q^{k-1} \sum_{a=1}^{q} \chi(a)\left(-\frac{k !}{(2 \pi i)^{k}} \sum_{\substack{t=-\infty \\ t \neq 0}}^{+\infty} \frac{e\left(\frac{a t}{q}\right)}{t^{k}}\right)=-\frac{k ! q^{k-1}}{(2 \pi i)^{k}} \sum_{\substack{t=-\infty \\ t \neq 0}}^{+\infty} \frac{G(t, \chi)}{t^{k}} .
$$

Therefore from Lemmas 2, 5 and 6, we may have

$$
\begin{aligned}
& \sum_{\substack{\chi \bmod _{\chi \neq \chi_{0}} \\
\chi \neq}}\left|C\left(m, n, r, \chi ; p^{\alpha}\right)\right|^{2}\left|B_{k, \chi}\right|^{2} \\
= & \frac{(k !)^{2} p^{2(k-1)^{\alpha}} \phi\left(p^{\alpha}\right)}{(2 \pi)^{2 k}} \sum_{\substack{\bmod \\
\chi \neq \chi_{0}}} \sum_{\substack{p^{\alpha}=-\infty \\
r_{1} \neq r_{2}=-\infty \\
r_{2} \neq 0}}^{+\infty} \frac{G\left(r_{1}, \chi\right) \overline{G\left(r_{2}, \chi\right)}}{\left(r_{1} r_{2}\right)^{k}} \\
+ & \frac{(k !)^{2} p^{2(k-1) \alpha}}{(2 \pi)^{2 k}} \sum_{a=2}^{p^{\alpha}} \sum_{b=1}^{p^{\alpha}} \in\left(\frac{m b^{r}\left(a^{r}-1\right)+n b(a-1)}{p^{\alpha}}\right)
\end{aligned}
$$




$$
\begin{aligned}
& \times \sum_{\substack{\bmod p^{\alpha} \\
\chi \neq \chi_{0}}} \chi(a) \sum_{\substack{r_{1}=-\infty \\
r_{1} \neq 0}}^{+\infty} \sum_{\substack{r_{2}=-\infty \\
r_{2} \neq 0}}^{+\infty} \frac{G\left(r_{1}, \chi\right) \overline{G\left(r_{2}, \chi\right)}}{\left(r_{1} r_{2}\right)^{k}} \\
= & \frac{2 \zeta(2 k)(k !)^{2} p^{(2 k+1) \alpha}}{(2 \pi)^{2 k}}+O\left(p^{\left(2 k+\frac{1}{2}\right) \alpha+\epsilon}\right) .
\end{aligned}
$$

This completes the proof of Theorem 2.

Now we come to prove Theorem 3. From Lemmas 2, 8 and 9, we also have

$$
\begin{aligned}
& \sum_{\substack{\bmod _{\chi \neq \chi_{0}} \\
\chi \neq p^{\alpha}}}\left|C\left(m, n, r, \chi ; p^{\alpha}\right)\right|^{2} \tau^{h}(\bar{\chi}) B_{k, \chi}^{h}
\end{aligned}
$$

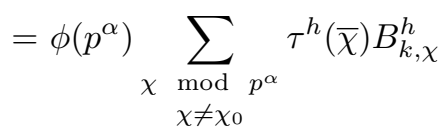

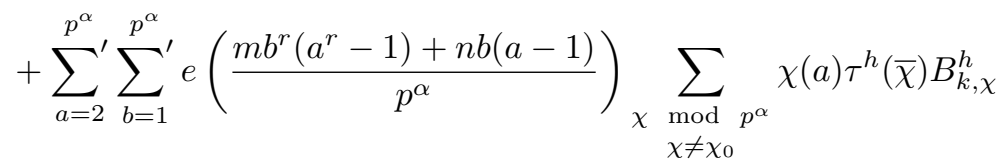

$$
\begin{aligned}
& =\frac{(-k !)^{h} p^{(k-1) h \alpha}}{(2 \pi i)^{k h}} \sum_{a=2}^{p^{\alpha}} \sum_{b=1}^{p^{\alpha}} \sum^{\prime} e\left(\frac{m b^{r}\left(a^{r}-1\right)+n b(a-1)}{p^{\alpha}}\right) \\
& \times \sum_{\chi \bmod p^{\alpha}}^{*} \chi(a) \tau^{h}(\bar{\chi})\left(\sum_{\substack{t=-\infty \\
t \neq 0}}^{+\infty} \frac{G(t, \chi)}{t^{k}}\right)^{h}+\phi\left(p^{\alpha}\right) \sum_{\substack{\bmod p^{\alpha} \\
\chi \neq \chi_{0}}}^{*} \tau^{h}(\bar{\chi}) B_{k, \chi}^{h} \\
& =: \frac{2^{h-1}(k !)^{h} p^{(k h+2) \alpha}}{(-1)^{(k-1) h}(2 \pi i)^{k h}}+O\left(p^{(k h+1) \alpha+\epsilon}\right)+E .
\end{aligned}
$$

Then for any primitive character $\chi$, from the property of Gauss sums that $G(t, \chi)=\bar{\chi}(t) \tau(\chi)$ and $\tau(\chi) \tau(\bar{\chi})=\chi(-1) p^{\alpha}$, we have

$$
\begin{aligned}
E= & \frac{(-k !)^{h} p^{(k-1) h \alpha}}{(2 \pi i)^{k h}} \sum_{a=2}^{p^{\alpha}} \sum_{b=1}^{p^{\alpha}} e\left(\frac{m b^{r}\left(a^{r}-1\right)+n b(a-1)}{p^{\alpha}}\right) \\
& \times \sum_{\chi \bmod p^{\alpha}}^{*} \chi(a) \tau^{h}(\bar{\chi})\left(\sum_{\substack{t=-\infty \\
t \neq 0}}^{+\infty} \frac{G(t, \chi)}{t^{k}}\right) \\
= & \frac{(-k !)^{h} p^{k h \alpha}}{(2 \pi i)^{k h}} \sum_{a=2}^{p^{\alpha}} \sum_{b=1}^{p^{\alpha}} e\left(\frac{m b^{r}\left(a^{r}-1\right)+n b(a-1)}{p^{\alpha}}\right)
\end{aligned}
$$




$$
\begin{aligned}
& \times \sum_{\chi \bmod p^{\alpha}}^{*} \chi^{h}(-1) \chi(a)\left(\sum_{\substack{t=-\infty \\
t \neq 0}}^{+\infty} \frac{\bar{\chi}(t)}{t^{k}}\right)^{h} \\
& =\frac{(-k !)^{h} p^{k h \alpha}}{(2 \pi i)^{k h}} \sum_{a=2}^{p^{\alpha}} \sum_{b=1}^{p^{\alpha}} e\left(\frac{m b^{r}\left(a^{r}-1\right)+n b(a-1)}{p^{\alpha}}\right) \\
& \times \sum_{\chi}^{*} \chi^{h}(-1) \chi(a)\left(1+\bar{\chi}(-1)(-1)^{k}\right)^{h} L^{h}(k, \bar{\chi}) \\
& = \begin{cases}\frac{(-2 k !)^{h} p^{k h \alpha}}{(2 \pi i)^{k h}} \sum_{a=2}^{p^{\alpha}} \sum_{b=1}^{p^{\alpha}} e\left(\frac{m b^{r}\left(a^{r}-1\right)+n b(a-1)}{p^{\alpha}}\right) & \\
\times \sum^{*} \chi(-1)=1 & \\
& \\
\frac{(2 k !)^{h} p^{k h \alpha}}{(2 \pi i)^{k h}} \sum_{a=2}^{p^{\alpha}} \sum_{b=1}^{\prime}(k, \bar{\chi}), & \text { if } 2 \mid k ; \\
\times \sum^{*} \chi(-1)=-1 & \\
& \\
& \end{cases}
\end{aligned}
$$

Therefore from Lemma 10, we may immediately obtain

$\sum_{\substack{\bmod p^{\alpha} \\ \chi \neq \chi_{0}}}\left|C\left(m, n, r, \chi ; p^{\alpha}\right)\right|^{2} \tau^{h}(\bar{\chi}) B_{k, \chi}^{h}=\frac{2^{h-1}(k !)^{h} p^{(k h+2) \alpha}}{(-1)^{(k-1) h}(2 \pi i)^{k h}}+O\left(p^{\left(k h+\frac{3}{2}\right) \alpha+\epsilon}\right)$.

This completes the proof of Theorem 3 .

Acknowledgments. The author expresses his gratitude to the referee for his/her very helpful and detailed comments.

\section{References}

[1] T. M. Apostol, Introduction to Analytic Number Theory, Springer-Verlag, New YorkHeidelberg, 1976.

[2] S. Chowla, On Kloosterman's sum, Norske Vid. Selsk. Forh. (Trondheim) 40 (1967), $70-72$.

[3] T. Cochrane and Z. Zheng, Bounds for certain exponential sums, Asian J. Math. 4 (2000), no. 4, 757-773.

[4] Upper bounds on a two-term exponential sum, Sci. China Ser. A 44 (2001), no. 8, 1003-1015.

[5] R. Dabrowski and B. Fisher, A stationary phase formula for exponential sums over $\mathbf{Z} / p^{m} \mathbf{Z}$ and applications to GL(3)-Kloosterman sums, Acta Arith. 80 (1997), no. 1, 1-48.

[6] H. Davenport, Multiplicative Number Theory, Springer-Verlag, New York-Berlin, 1980.

[7] H. Darvenport and H. Heibronn, On an exponential sum, Proc. London Math. Soc. 41 (1936), 449-453.

[8] L. K. Hua, On exponential sums, Sci. Record (N.S.) 1 (1957), 1-4.

[9] Introduction to Number Theory, Science Press, Beijing, 1979.

[10] H. Liu and W. Zhang, On the hybrid mean value of Gauss sums and generalized Bernoulli numbers, Proc. Japan Acad. Ser. A Math. Sci. 80 (2004), no. 6, 113-115. 
[11] _ Hybrid mean value of generalized Bernoulli numbers, general Kloosterman sums and Gauss sums, J. Korean Math. Soc. 44 (2007), no. 1, 11-24.

[12] H. Liu and X. Zhang, On the mean value of $\frac{L^{\prime}}{L}(1, \chi)$, J. Math. Anal. Appl. 320 (2006), no. $2,562-577$.

[13] J. H. Loxton and R. A. Smith, On Hua's estimate for exponential sums, J. London Math. Soc. (2) 26 (1982), no. 1, 15-20.

[14] J. H. Loxton and R. C. Vaughan, The estimate for complete exponential sums, Canada Math. Bull. 26 (1995), no. 4, 442-454.

[15] A. V. Malyšhev, A generalization of Kloosterman sums and their estimates, Vestnik Leningrad. Univ. 15 (1960), no. 13, 59-75.

[16] C. L. Siegel, Über die Classenzahl quadratischer Zahlkörper, Acta Arith. 1 (1935), 83-86.

[17] R. A. Smith, Estimates for exponential sums, Proc. Amer. Math. Soc. 79 (1980), no. 3, 365-368.

[18] A. Weil, On some exponential sums, Proc. Nat. Acad. Sci. U. S. A. 34 (1948), 204-207.

[19] Z. Xu, T. Zhang, and W. Zhang, On the mean value of the two-term exponential sums with Dirichlet characters, J. Number Theory 123 (2007), no. 2, 352-362.

[20] Y. Ye, Estimation of exponential sums of polynomials of higher degrees. II, Acta Arith. 93 (2000), no. 3, 221-235.

[21] W. Zhang, On a Cochrane sum and its hybrid mean value formula, J. Math. Anal. Appl. 267 (2002), no. 1, 89-96.

$[22] \ldots$ On the general Kloosterman sum and its fourth power mean, J. Number Theory 104 (2004), no. 1, 156-161.

[23] _ On the fourth power mean of the general Kloosterman sums, Indian J. Pure Appl. Math. 35 (2004), no. 2, 237-242.

[24] W. Zhang, Y. Yi, and X. He, On the $2 k$-th power mean of Dirichlet L-functions with the weight of general Kloosterman sums, J. Number Theory 84 (2000), no. 2, 199-213.

College of Mathematics and Information Science

ShaANXI NORMAL University

Xi'An, ShaAnXi, P. R. China

E-mail address: tpzhang@snnu.edu.cn 